\title{
Applications of the Hurwitz-Lerch zeta-function
}

\author{
Tomihiro Arai ${ }^{1}$, Kalyan Chakraborty ${ }^{2}$, Jing $\mathrm{Ma}^{3,}$ * \\ ${ }^{1}$ Grad. School of Advances Tech. Kinki Univ.,Iizuka, Japan \\ ${ }^{2}$ Harish-Chandra Institute, Allahabad, Allahabad, India \\ ${ }^{3}$ Dept. of Math., Jilin Univ., Changchun, PRC
}

Email address:

kalychak@gmail.com (K. Chakraborty),jma@jlu.edu.cn (Jing Ma)

\section{To cite this article:}

Tomihiro Arai, Kalyan Chakraborty, Jing Ma. Applications of the Hurwitz-Lerch Zeta-Function. Pure and Applied Mathematics Journal. Special Issue: Abridging over Troubled Water---Scientific Foundation of Engineering Subjects. Vol. 4, No. 2-1, 2015, pp. 30-35.

doi: $10.11648 /$ j.pamj.s.2015040201.16

\begin{abstract}
In this paper, we shall exhibit the use of two principles, "principle of decomposition into residue classes" and "binomial principle of analytic continuation" due to Ram Murty and Sinha and indicate a certain distribution property and the functional equation for the Lipschitz-Lerch transcendent at integral arguments ofs. By considering the limiting cases $s \rightarrow n$, we can also deduce new striking identities for Lipschizt-Lerch transcendent, among which is the Gauss second formula for the digamma function, Lipschitz-Lerch transcendent
\end{abstract}

Keywords: Decomposition into Residue Classes, Binomial Expansion, Distribution Property, Zeta-Functions, Functional Equation

\section{Introduction}

In1882, Hurwitz [5] defined the perturbed Riemann zeta-function, $\zeta(s, x)$, by the Dirichlet series

$$
\zeta(s, x)=\sum_{n=0}^{\infty} \frac{1}{(n+x)^{s}},
$$

for any $x$ satisfying $0<x \leq 1$. This function is defined in the first instance for $\sigma=\operatorname{Re} s>1$ and then can be continued meromorphically over the whole plane with a simple pole at $s=1$ with residue 1 . The special case $\zeta(s, 1)=\zeta$ (s) was introduced by Riemann [13] in1859 in his celebrated and unique paper on the distribution of primes that now bears Riemann's name. He indicated how it can be used to study the distribution of primes. In his study of $\zeta(s, x)$, Hurwitz was motivated by the problem of analytic continuation of Dirichlet $L$-functions. For any Dirichlet character $\chi(\bmod q)$, we may write

$$
L(s, \chi):=\sum_{n=o}^{\infty} \frac{\chi(n)}{n^{s}}=q^{-s} \sum_{a=1}^{q} \chi(a) \zeta\left(s, \frac{a}{q}\right),
$$

and therefore the analytic continuation of the Hurwitz zeta function gives us the same for the $L(s, \chi)$. Thus, Hurwitz confined his attention to $x$ rationals lying in the interval $(0,1)$. A globally convergent series for $s \neq 1$ was given by Helmut Hasse in 1930 [4] and rediscovered by Sondow [14] (cf. [17] for further details):

$$
\zeta(s, x)=\frac{1}{s-1} \sum_{n=0}^{\infty} \frac{1}{(n+1)} \sum_{k=0}^{n}(-1)^{k}\left(\begin{array}{l}
n \\
k
\end{array}\right)(x+k)^{1-s}, x>-1,1 \neq s \in C,
$$

hence it follows that at $s=1$ it has a simple pole with residue 1 , the constant term being given by

$$
\lim _{s \rightarrow 1}\left[\zeta(s, a)-\frac{1}{s-1}\right]=-\frac{\Gamma^{\prime}(a)}{\Gamma(a)}=-\psi(a),
$$

where $\Gamma$ is the gamma function and $\psi$ is the digamma function.

Let $\mathbf{C}$ be the complex plane, and let

$$
\begin{gathered}
\mathbf{C}_{1}:=\mathbf{C} \backslash[1,+\infty), \mathbf{C}_{2}:=\{x: \operatorname{Re} x>0\}, \\
\mathbf{C}_{3}:=\mathbf{C} \backslash\{0,-1,-2, \cdots\} .
\end{gathered}
$$

We introduce the Hurwitz-Lerch zeta-function $\Phi(z, s, x)$ defined by Erdélyiet al in [3] originally as 


$$
\Phi(z, s, x)=\sum_{n=0}^{\infty} \frac{z^{n}}{(n+x)^{s}}, s, z \in \mathbf{C},|z|<1, x \in \mathbf{C}_{3} .
$$

In [7, p.5 Theorem 1], the function $\Phi(z, s, x)$ extends to an analytic function in three variables $z, s, x$ for

$$
z \in \mathbf{C}_{1}, x \in \mathbf{C}_{2}
$$

and $s \in \mathbf{C}$ or $s \in \mathbf{C} \backslash\{1\}$ according as $z \neq 1$ or $z=1$, by the

contour integral representation

$$
\begin{gathered}
\Phi(z, s, x)=-\frac{\Gamma(1-s)}{2 \pi i} \int_{\infty}^{(+0)} \frac{(-t)^{s-1} e^{-x t}}{1-z e^{-t}} d t, \\
x \in \mathbf{C}_{2},|\arg (-t)| \leq \pi .
\end{gathered}
$$

The contour starts at $\infty$, encircles the origin once counter-clockwise and returns to its starting point. The initial and final values of $\arg (-t)$ are $-\pi$ and $\pi$ respectively.

The most general distribution property of the Hurwitz-Lerch zeta-function follows from the principle of decomposition into residue classes:

$$
\Phi(z, s, x)=q^{-s} \sum_{j=0}^{q-1} \Phi\left(z^{q}, s, \frac{x+j}{q}\right) z^{j} .
$$

The uniformization of $\Phi(z, s, x)$ is called the Lipschitz-Lerch transcendent and denoted by

$$
L(z, s, a)=\sum_{n=0}^{\infty} \frac{e^{2 \pi i n z}}{(n+a)^{s}}=\Phi\left(e^{2 \pi i z}, s, a\right),
$$

which generalizes not only the Hurwitz zeta-function

$$
\zeta(s, a)=L(1, s, a)
$$

but also the polylogarithm function (or the Lerch zeta-function)

$$
l_{s}(x)=L(x, s, 1)=\sum_{n=1}^{\infty} \frac{e^{2 \pi i n x}}{n^{s}} .
$$

Recently, Li-Hashimoto-Kanemitsu [10] applied the method of finite Fourier series to elucidate the existing results on the Lipschitz-Lerch transcendent. The general distribution property of the Lipschitz-Lerch transcendent (used in Srivastava's first method) is a special case of (1.5)

$$
L(z, s, a)=q^{-s} \sum_{j=0}^{q-1} L\left(q z, s, \frac{a+j}{q}\right) e^{2 \pi i j z}
$$

$([15,(15), \quad p .339])$. The functional equation for the Lipschitz-Lerch transcendent reads ([15, (29), p. 125])

$$
\begin{aligned}
& L(x, 1-s, a)=\frac{\Gamma(s)}{(2 \pi)^{s}} \\
& \times\left\{e^{\pi i\left(\frac{1}{2} s-2 a x\right)} L(-a, s, x)+e^{-\pi i\left(\frac{1}{2} s-2 a(1-x)\right)} L(a, s, 1-x)\right\} .
\end{aligned}
$$

It has turned out that it is a combination of these two ingredients that captures the whole spectrum and gives an immediate proof of new intriguing limiting relations. It also gives all those types of linear combination expressions for a class of zeta-functions and as a special case for polynomials. However, we can make full use of intrinsic properties of the Hurwitz-Lerchzeta-function at integral arguments $s$ instead to recover somewhat more general results with more ease.

In Section 2, we shall apply Murty-Sinha's method encoded in [12, Theorem3.1] as the binomial principle of analytic continuation to recover the continuation of $\Phi(z, s, x)$ from the polylogarithm $\operatorname{Li}_{s}(z)$ which is defined by

$$
\operatorname{Li}_{s}(z)=z \Phi(z, s, 1)=\sum_{n=1}^{\infty} \frac{z^{n}}{n^{s}}, \sigma=\operatorname{Re} s>1 .
$$

In fact, the derivation not only gives the analytic continuation of $\mathrm{Li}_{s}(z)$, but also gives the analytic continuation of various Dirichlet series, such as $l_{s}(x)$, $\zeta(s, x), L(z, s, a)$ and $L(s, \chi)$ etc.

In Section 3, we show the distribution property of the Hurwitz-Lerchzeta and Lipschitz-Lerch transcendent at integral $\operatorname{arguments} n$, in the sense of $s \rightarrow n, \quad n \in \mathbf{N}$. Some known results via Hurwitz-Lerch zeta-function will be illustrated in Section 4. In Section 5, we give an illustrative example.

\section{Analytic continuation of the Hurwitz-Lerchzeta-function revisited}

In this section, we shall recover the analytic property of the Hurwitz-Lerch zeta-function in the spirit of Murty-Sinha's [12, Theorem 3.1] and give an elementary proof of the analytic property of the polylogarithm function $\mathrm{Li}_{s}(z)$, where we use the equation (2.4) (below) corresponding to (1.2) and(1.9).

Theorem 1. ([7, Theorem 3.1])

The Hurwitz-Lerch zeta-function $\Phi(z, s, x)$ defined by (1.4) can be analytically continued to a meromorphic function in three complex variables $z, s, x$ for $z \in \mathbf{C}_{1}, x \in \mathbf{C}_{2}$; $s \in \mathbf{C}$ or $s \in \mathbf{C} \backslash\{1\}$ according as $z \neq 1$ or $z=1$, and the special case $\Phi(1, s, 1)=\zeta(s)$ has a simple pole with residue 1 .

Proof. Without loss of generality, we may suppose that $0<|x|<1$. (If not, we may begin our summation of the 
series from $n_{0}$ with $n_{0}>|x|$ ).For $\operatorname{Re} s>1$, we write $\Phi(z, s, x)$ as

$$
\Phi(z, s, x)=x^{-s}+\sum_{n=1}^{\infty} \frac{z^{n}}{n^{s}}\left(1+\frac{x}{n}\right)^{-s}
$$

expand the summand by the binomial theorem and interchange summations to get

$$
\Phi(z, s, x)-\mathrm{Li}_{s}(z)-x^{-s}=\sum_{r=1}^{\infty}\left(\begin{array}{c}
-s \\
r
\end{array}\right) \operatorname{Li}_{s+r}(z) x^{r} .
$$

Clearly, for sufficiently large $r, \mathrm{Li}_{s+r}(z)$ is bounded. Applying ther-throot test, together with the observation

$$
\log \left|\left(\begin{array}{c}
-s \\
r
\end{array}\right)\right| \leq \sum_{j=1}^{r} \log \left(1+\frac{|s|}{j}\right) \ll|s| \log r
$$

shows that the series converges absolutely for $|x|<1$ and $\operatorname{Re} s>1$ since $\mathrm{Li}_{s+r}(z)$ is absolutely convergent there. The sum from $r=0$ to infinity being a moromorphic function, we deduce that $\Phi(z, s, x)$ has a meromorphic continuation for Re $s>0$.

The second part of the assertion is trivial since the possible poles of $\Phi(z, s, x)$ can only occur among the integer translates of the poles of $\mathrm{Li}_{s}(z)$, completing the proof.

It is to be noted that the expansions of this kind were also discovered by many authors, for instance, readers may refer to [4], [14].

Applying the method encoded in Theorem 1, we may show the analytic continuation of $\mathrm{Li}_{s}(z)$.

Putting $x=\frac{1}{2}$ in (2.1) and noting that for $|z|<1$

$$
\begin{aligned}
z \Phi\left(z^{2}, s, \frac{1}{2}\right) & =2^{s} \sum_{n=0}^{\infty} \frac{z^{2 n+1}}{(2 n+1)^{s}} \\
& =2^{s}\left(\sum_{n=1}^{\infty} \frac{z^{n}}{n^{s}}-\sum_{n=1}^{\infty} \frac{z^{2 n}}{(2 n)^{s}}\right)=2^{s} \operatorname{Li}_{s}(z)-\operatorname{Li}_{s}\left(z^{2}\right)
\end{aligned}
$$

we have

Proposition 1. We have

$$
2^{s} \operatorname{Li}_{s}(z)=2^{s} z+(z+1) \operatorname{Li}_{s}\left(z^{2}\right)+z \sum_{r=1}^{\infty} 2^{-r}\left(\begin{array}{c}
-s \\
r
\end{array}\right) \operatorname{Li}_{s+r}\left(z^{2}\right) \text {. }
$$

Eq. (2.2) gives the analytic continuation of $\mathrm{Li}_{s}(z)$ by induction. For clearly, it is valid in the half-plane $\operatorname{Re} s>1$ of absolute convergence and it allows us to obtain inductively a meromorphic continuation of $\mathrm{Li}_{s}(z)$.

Furthermore, putting $x=\frac{a}{q}(0<a<q)$,

(2.1) gives an analytic continuation of $\Phi\left(z, s, \frac{a}{q}\right)-\mathrm{Li}_{s}(z)$ for $\operatorname{Re} s>0$, with possible poles in the set

$$
S_{q}:=\left\{s \in \mathbf{C} \mid s=1+\frac{2 \pi i m}{\log q}, m \in \mathbf{Z}\right\} .
$$

Rewriting (1.4) as (1.5), putting $x=1$ in (1.5) and incorporating (1.11), we have

Proposition 2. We have

$$
\left(q^{s}-q\right) \operatorname{Li}_{s}(z)=\sum_{j=1}^{q}\left[\Phi\left(z^{q}, s, \frac{j}{q}\right) z^{j}-\mathrm{Li}_{s}(z)\right]
$$

Taking $q=2,3$, we find that $\operatorname{Li}_{s}(z)$ extends analytically for Re $s>0$ except for a possible pole at $S_{2} \cap S_{3}$, but the only element is 1 in the intersection $S_{2} \cap S_{3}$. Now, we consider $\operatorname{Li}_{1}(z)$ for $|z|<1$ and $|z|=1$ separately. Since $\operatorname{Li}_{1}(z)=-\log (1-z)$ is analytic in the region $|z|<1$, the remainder follows from $|z|=1$ and (2.2) gives an analytic continuation of $\left(2^{s}-2\right) \mathrm{Li}_{s}(z)$ for $\operatorname{Re} s>0$. A simple calculation shows that

$$
\lim _{s \rightarrow 1}(s-1) \operatorname{Li}_{s}(z)= \begin{cases}1, & z=1 \\ 0, & z \neq 1,|z|=1 .\end{cases}
$$

Combining this with (2.2) and using induction, we immediately deduce

Theorem 2.The polylogarithm $\mathrm{Li}_{s}(z)$ defined by (1.11) can be extended to an analytic function for $s \in C$ or $s \in C \backslash\{1\}$ according as $z \neq 1$ or $z=1$, in the special case $s=1, z=1$ it has a simple pole with residue 1 .

\section{The distribution properties of Lipschitz-Lerchtranscendent at integral arguments}

The value of the Hurwitz-Lerch zeta-function $\Phi(a, s, a)$ at $s=-N$ is given by

$$
\Phi(z,-N, a)=\sum_{r=0}^{N} \sum_{n=r}^{N}\left(\begin{array}{l}
N \\
n
\end{array}\right) \frac{r ! z^{r} a^{N-n} S(n, r)}{(1-z)^{r+1}}
$$

in $[7$, p.14, Theorem 6], where $S(n, r)$ are the Stirling numbers of the second kind, or by

$$
\mathrm{B}_{N+1}(a, z)=-(N+1) \Phi(z,-N, a), 0<a,
$$


in $[15$, p.126,(40)], where $|z| \leq 1, z \neq 1, N=0,1,2, \cdots$ and $B_{N}(a, z)$ denote the Lipschitz-Lerch-Bernoulli functions defined by

$$
\frac{t e^{a t}}{z e^{t}-1}=\sum_{N=0}^{\infty} \mathrm{B}_{N}(a, z) \frac{t^{N}}{N !}, \quad|t+\log z|<2 \pi .
$$

In this section, we shall give some distribution properties of the Lipschitz-Lerch transcendent at integral arguments $s$, in the sense of $s \rightarrow N$.

Let $i=\sqrt{-1}, \omega_{q}{ }^{j}=e^{2 \pi i j / q},(j \in N, 0 \leq j<q)$, we recall the inverse formula of (1.5) (cf. T. Nakamura[11, Theorem 2.2])

$$
\Phi\left(z, s, \frac{x+j}{q}\right)=q^{s-1} z^{-j / q} \sum_{k=0}^{q-1} \omega_{q}^{-j k} \Phi\left(\omega_{q}^{k} z^{1 / q}, s, x\right)
$$

where $x, s$ and $z$ satisfy the conditions

$$
x>0, z \in \mathbf{C}_{1},-\frac{\pi}{q}<\arg z^{1 / q} \leq \frac{\pi}{q}
$$

and $s \in \mathbf{C}$ or $s \in \mathbf{C} \backslash\{1\}$ according as $z \neq 1$ or $z=1$.

This follows from the finite Fourier transform pairs as follows.

Any $\operatorname{sum} S$ over a residue class $j \equiv k(\bmod q)$ may be expressed as

$$
S=\sum_{j \equiv k(\bmod q)} a_{j}=\sum_{j} \chi_{k}(j) \mathrm{a}_{j}
$$

where $\chi_{k}=\chi_{k, q}$ is the characteristic function of the set of residue class $j \equiv k(\bmod q)$. By [8, (4.22),p.74]

$$
\chi_{k, q}=\frac{1}{q} \sum_{\ell(\bmod q)} \overline{\varepsilon_{k}(\ell)} \varepsilon_{\ell}
$$

where $\varepsilon_{\ell}(j)=e^{2 \pi i(\ell / q) j}$. Hence

$$
S=\frac{1}{q} \sum_{\ell(\bmod q)} \overline{\varepsilon_{k}(\ell)} \sum_{j} \varepsilon_{\ell}(\mathrm{j}) a_{j}
$$

Note that

$$
\Phi\left(z, s, \frac{x+j}{q}\right)=q^{s} z^{-j / q} S_{j}
$$

where $S_{j}=\sum_{k \equiv j} a_{k}$ with $a_{k}=\frac{\left(z^{1 / \mathrm{q}}\right)^{k}}{(k+s)^{s}}$. Hence by (3.5),

$$
\Phi\left(z, s, \frac{x+j}{q}\right)=q^{s} z^{-j / q} \frac{1}{q} \sum_{k(\bmod q)} \overline{\varepsilon_{j}(k)} \sum_{\ell} \varepsilon_{k}(\ell) a_{\ell},(3.7)
$$

the inner sum being $\Phi\left(\omega^{k} z^{1 / q}, s, x\right)$, (3.2) follows.

The combination of (1.5) (or (3.2)) and (1.10) leads to

Theorem 3.For $1<q \in \mathbf{Z}$ and $0<x<1$, $\operatorname{Re} a>0$, we have

$$
\begin{aligned}
& q^{n-1} \sum_{j=0}^{q-1} L\left(q x, 1-n, \frac{a+j}{q}\right) e^{2 \pi i j x}=\frac{\Gamma(n)}{(2 \pi)^{n}} \\
& \times\left\{e^{\pi i\left(\frac{n}{2}-2 a x\right)} L(-a, n, x)+e^{-\pi i\left(\frac{n}{2}-2 a(1-x)\right)} L(a, n, 1-x)\right\},
\end{aligned}
$$

$1<n \in \mathbf{N}$.

Theorem 4.For $1<q \in \mathbf{Z}$ and $q>p \in \mathbf{N}$, we have

$$
\begin{aligned}
& q^{n-1} \sum_{j=1}^{q} e^{2 \pi i j p / q} L\left(1,1-n, \frac{j}{q}\right)=i \frac{\Gamma(n)}{(2 \pi)^{n}} \\
& \times\left\{e^{-\frac{n \pi i}{2}} L\left(1, n, \frac{p}{q}\right)-e^{\frac{n \pi i}{2}} L\left(1, n, 1-\frac{p}{q}\right)\right\}, 1<n \in N .
\end{aligned}
$$

Theorem 5. The limiting case $s \rightarrow 1$ of

$$
\sum_{j=1}^{q-1} e^{-2 \pi i j \frac{p}{q}} L\left(\frac{j}{q}, s, 1\right)=q^{1-s} L\left(1, s, \frac{p}{q}\right)-L(1, s, 1)
$$

implies Gauss's second formula for the digamma function $\psi\left(\frac{p}{q}\right)=-\gamma-\log q-\frac{\pi}{2} \cot \frac{p}{q} \pi+2 \sum_{k \leq \frac{\pi}{2}} \cos \frac{2 p k}{q} \pi \log \left(2 \sin \frac{k}{q}\right)$.

The limit $s \rightarrow n$ gives similar identities.

\section{Gamma, Psi, Bernoulli function via the Hurwitz-Lerch zeta-function}

The functional equation relates the values of the zeta-function in the left and right-hand sides of the complex plane. For integers $1 \leq m<n$,

$$
\zeta\left(1-s, \frac{m}{n}\right)=\frac{2 \Gamma(\mathrm{s})}{(2 \pi n)^{s}} \sum_{k=1}^{n} \cos \left(\frac{\pi s}{2}-\frac{2 \pi k m}{n}\right) \zeta\left(s, \frac{k}{n}\right) .
$$

Catalan's constant $G$ is defined by

$$
G=\sum_{n=1}^{\infty} \frac{(-1)^{n}}{(2 n-1)^{2}}
$$

This is the special value of Dirichlet $L$-function with the primitive character $\chi_{-4}$ associated to the Gaussian field $Q(\sqrt{-4})$ :

$$
C l_{2}\left(\frac{\pi}{2}\right)=G=L\left(2, \chi_{-4}\right)=\sum_{n=1}^{\infty} \frac{\chi_{-4}(n)}{n^{2}}
$$

and on $[15, \mathrm{p} .106]$ we have 


$$
C l_{2}(x)=x \log \pi-x \log \sin \frac{x}{2}-2 \pi \log \frac{G\left(1+\frac{x}{2 \pi}\right)}{G\left(1+\frac{x}{2 \pi}\right)},
$$

where $G(x)$ is the $G$-function. Use of this would make the

$$
\begin{aligned}
& q^{-s} \sum_{j=0}^{q-1} L\left(q x, s, \frac{a+j}{q}\right) e^{2 \pi i j x}=L(x, s, a)=\frac{\Gamma(1-s)}{(2 \pi)^{1-s}} \\
& \times\left\{e^{\pi i\left(\frac{1}{2}(1-s)-2 a x\right)} L(-a, 1-s, x)+e^{-\pi i\left(\frac{1}{2}(1-s)-2 a(1-x)\right)} L(a, 1-s, 1-x)\right\}
\end{aligned}
$$

except at the singularities of $L$, in which case, the identity is to mean the limit.

Specializing the parameter a suitably, Theorem 6 gives rise to all the known partial formulas.

\section{An Example}

We shall illustrate the above situation by the following concrete example. Put $m=q r+k$ with $r=0,1, \cdots, \infty$, $k=0,1, \cdots,(q-1)$, with $r$ and $k$ not simultaneously zero. Then

$$
\begin{aligned}
& l_{s}\left(\frac{p}{q}\right)=\sum_{n=0}^{\infty} \frac{e^{2 \pi i m p / q}}{m^{s}} \\
& =\sum_{r=0}^{\infty} \sum_{k=0}^{q-1} \frac{e^{2 \pi i(q r+k) p / q}}{(q r+k)^{s}}=q^{-s}\left(\sum_{r=1}^{\infty} \frac{1}{r^{s}}+\sum_{r=0}^{\infty} \sum_{k=1}^{q-1} e^{2 \pi i k p / q} \zeta\left(s, \frac{k}{q}\right)\right)
\end{aligned}
$$

Ishibashi [6] was the first who gave a computational proof of the generalized inverse Eisenstein formula

$$
\sum_{j=1}^{q} e^{2 \pi i \frac{j}{q} p} \zeta\left(\mathrm{s}, \frac{j}{q}\right)=k^{s-1} l_{s}\left(\frac{p}{q}\right)
$$

for all $s=n \in N$, and referred to the generalized Eisenstein formula (with a slight misstatement corrected)

$$
\sum_{k=1}^{q} e^{-2 \pi \frac{p}{q} k} l_{s}\left(\frac{k}{q}\right)=q^{1-s} \zeta\left(\mathrm{s}, \frac{p}{q}\right),
$$

valid for all complex $s \neq 1$, as another formula implying the formula (5.2)with $l_{s}(0)=\zeta(s)=\zeta(s, 1)$ being the Riemann zeta-function or

$$
\sum_{k=1}^{q-1} e^{-2 \pi i \frac{a}{q} k} l_{s}\left(\frac{k}{q}\right)=q^{1-s} \zeta\left(s, \frac{a}{q}\right)-\zeta(s)
$$

and the generalized inverse Eisenstein formula

$$
\sum_{k=1}^{q} e^{2 \pi i \frac{k}{q} p} \zeta\left(s, \frac{k}{q}\right)=q^{s-1} l_{s}\left(\frac{p}{q}\right)
$$

are the genuine generalizations of the Eisenstein and the inverse Eisenstein formula and not only represent a relation between the two bases

$$
\left\{l_{s}\left(\frac{a}{q}\right) \mid a=0, \cdots, q-1\right\}
$$

and

$$
\left\{\zeta\left(s, \frac{a}{q}\right) \mid a=0, \cdots, q-1\right\} .
$$

For $1<q \in \mathbf{Z}$ and $q>p \in \mathbf{N}$, we have the identities

$$
\begin{aligned}
& q^{-s} \sum_{a=1}^{q} e^{2 \pi i \frac{a}{q} p} \zeta\left(s, \frac{a}{q}\right)\left(=l_{s}\left(\frac{p}{q}\right)=\right) \\
& =i \frac{\Gamma(1-s)}{(2 \pi)^{1-s}}\left\{e^{-\frac{\pi i s}{2}} \zeta\left(1-s, \frac{p}{q}\right)-e^{\frac{\pi i s}{2}} \zeta\left(1-s, 1-\frac{p}{q}\right)\right\},
\end{aligned}
$$

and

$$
\begin{aligned}
& \sum_{a=1}^{q} e^{-2 \pi i \frac{a}{q} p} l_{s}\left(\frac{a}{q}\right)\left(=q^{1-s} \zeta\left(s, \frac{p}{q}\right)=\right) \\
& =q^{1-s} \frac{\Gamma(1-s)}{(2 \pi)^{1-s}}\left\{e^{\frac{\pi i s}{2}} l_{1-s}\left(\frac{p}{q}\right)-e^{-\frac{\pi i s}{2}} l_{1-s}\left(1-\frac{p}{q}\right)\right\},
\end{aligned}
$$

where, for non-negative integer values of $s=n$, say, (5.6) and (5.7) are to mean the limit as $s \rightarrow n$.

\section{References}

[1] K. Chakraborty, S. Kanemitsu, and H. -L. Li, On the values of a classof Dirichlet series at rational arguments, Proc. Amer. Math. Soc. 138(2010), 1223-1230.

[2] G. Eisenstein, Aufgaben und Lehrs"atze, J. Reine Angew. Math. 27 (1844), 281-283=Math. Werke, Vol. 1, 1975, Chelsea, 108-110.

[3] A. Erdléyi, W. Magnus, F. Oberhettinger, and F. G. Tricomi, Highertrancendental functions vol 1, McGraw-hill, New York 1953.

[4] H. Hasse, EinSummierungsverfahrenf ur die Riemannsche ל-Reihe,Math. Z. 32(1930), 458-464.

[5] A. Hurwitz, MathematischeWerke, Vol. 2, Basel, Birkh“auser, 1932. 
[6] M. Ishibashi, An elementary proof of the generalized Eisenstein formula, Sitzungsber. Osterreich. Wiss. Wien, Math. -naturwiss. K1. 197 (1988), 443-447.

[7] S. Kanemitsu, M. Katsurada, and M. Yoshimoto, On the Hurwitz-Lerchzeta function, Aequationes Math. 59 (2000), $1-19$.

[8] S. Kanemitsu and H. Tsukada, Vistas of special functions, World Scientific,Singapore-London-New York. 2007.

[9] D. Klusch, On the Taylor expansion for the Lerch zeta function, J. Math.Anal. Appl. 170 (1992), 513-523.

[10] H. -L. Li, M. Hashimoto and S. Kanemitsu, On structural elucidationof Eisenstein's formula, Sci. China. 53 (2010), 2341-2350.

[11] T. Nakamura, Some topics related to Hurwitz-Lerch zeta functions, TheRamanujan J. 21 (2010), 285-302.

[12] M. Ram Murty and Kaneenika Sinha, Multiple Hurwitz zeta functions,Proc. Sympos. Pure Math.75 (2006), 135-156.
[13] B. Riemann, "Uber die Anzahl der Primzahlen, untereinergegebenenGr"osse, Monatsber. Akad. Berlin, (1859), 1-680 =Collected Works ofBernhard Riemann, ed. by $\mathrm{H}$. Weber, 2nd ed. Dover, New York 1953.

[14] J. Sondow, Analytic continuation of Riemann's zeta function and valuesat negative integers via Euler's transformation of series, Proc. Amer.Math. Soc. 120 (1994), 421-424.

[15] H. M. Srivastava and J. -S. Choi, Series associated with the Zeta and relatedfunctions, Kluwer Academic Publishers, Dordrecht-Boston-London2001.

[16] H. M. Stark, Dirichlet's class-number formula revisited, Contemp. Math.143 (1993), 571-577.

[17] X.-H. Wang, Analytic continuation of the Riemann zeta-function, toappear.

[18] J. R. Wilton, A proof of Burnside's formula for $\log \Gamma(x+1)$ and certainallied properties of Riemann's $\zeta$-function, Mess. Math.55 (1922/1923),90-93. 